\title{
Gigahertz Parabolic Pulse Generation in a High-Power Polarization- Maintaining Fiber Amplifier Seeded by a Passively Mode-locked VECSEL
}

\author{
P. Dupriez (1), K. G. Wilcox (2), C. Finot (1), A. Malinowski (1), J. K. Sahu (1), D. J. Richardson (1), \\ A. C. Tropper (2), and J. Nilsson (1) \\ 1: Optoelectronics Research Centre, University of Southampton, Southampton SO17 1BJ, U.K. \\ pad@orc.soton.ac.uk \\ 2: School of Physics and Astronomy, University of Southampton, Southampton SO17 1BJ, U.K. \\ act@phys.soton.ac.uk
}

\begin{abstract}
Fiber amplification of sub-picosecond pulses at $1.1 \mathrm{GHz}$ produced by a passively mode-locked VECSEL enabled the generation of parabolic pulses with average power exceeding $50 \mathrm{~W}$. Clean pulse compression down to 110 fs was demonstrated.
\end{abstract}

\begin{abstract}
Introduction
Gigahertz repetition rate femtosecond lasers at $1 \mu \mathrm{m}$ have generated large interest due to their potential use in many industrial and scientific applications as well as in telecommunications [1]. Ytterbium-doped fibers are attractive for high-power short-pulse sources in Master Oscillator Power Amplifier (MOPA) configurations thanks to the remarkable efficiency, broad gain bandwidth, and excellent thermal properties of $\mathrm{Yb}$-doped fibers. Furthermore, in the parabolic amplification regime, self-phase modulation (SPM) in the fiber amplifier lead to the generation of spectrally broad linearly chirped pulses that can be compressed to even shorter pulse duration [2]. To date, however this attractive approach has only been used with high energy pulses at repetition rates below $100 \mathrm{MHz}[3,4]$.
\end{abstract}

Here we report on the generation of parabolic pulses with gigahertz repetition rate. A sub-picosecond passively mode-locked optically-pumped verticalexternal-cavity surface-emitting semiconductor laser (VECSEL) is used as a seed source for a Yb-doped fiber MOPA system. In contrast to our previous work [5], in which the 300 fs pulses we obtained had a significant pedestal resulting from a nonlinear chirp, we are now much more successful in reaching the parabolic amplification regime. Thus we obtain a clean linear chirp and a much reduced pedestal, as well as significantly shorter pulses following compression in a pair of gratings. Pulses shorter than 110 fs were generated with average powers exceeding $50 \mathrm{~W}$ at $1.1 \mathrm{GHz}$ repetition rate. To the best of our knowledge, this result represents the first demonstration of high-power parabolic pulse amplification at gigahertz repetition rate.

\section{Experimental set-up}

The MOPA system is shown schematically in figure 1. The master oscillator is a passively mode-locked VECSEL emitting nearly transform-limited 0.5 ps pulses at $1.1 \mathrm{GHz}$ repetition rate with $20 \mathrm{~mW}$ average power at $1043 \mathrm{~nm}$. A similar VECSEL is described in [6]. The VECSEL seeds two cladding-pumped ytterbium doped fiber amplifiers (YDFAs). The first amplifier used a $2 \mathrm{~m}$ long double-clad ytterbium doped fiber (YDF) from Coractive (HPA-Yb-20-02) with core and cladding diameters of 20 and $128 \mu \mathrm{m}$ respectively. Forward pumped by a fiber-pigtailed laser diode at $915 \mathrm{~nm}$, the short fiber amplifier ensured efficient watt-level amplification with insignificant nonlinear distortions and minimal linear dispersion. After transmission through a polarizing high-power isolator, the amplified pulses with $1.3 \mathrm{~W}$ average power and 0.8 ps duration were launched into the second YDFA. This included an $8 \mathrm{~m}$-long polarization maintaining double-clad YDF fabricated at the ORC, with a mode field diameter of $16 \mu \mathrm{m}$, a birefringence of $3.5 \times 10^{-4}$ and a D-shaped cladding of $400 \mathrm{\mu m}$ in diameter. At high repetition rate, the required input energy to reach parabolic pulse regime [7] imposes watt-level average power which is also sufficient to saturate the high-power amplifier. The final stage was pumped from the signal output end by a diode stack source emitting around $973 \mathrm{~nm}$. A fraction of the power was extracted for grating compression and autocorrelation measurements.

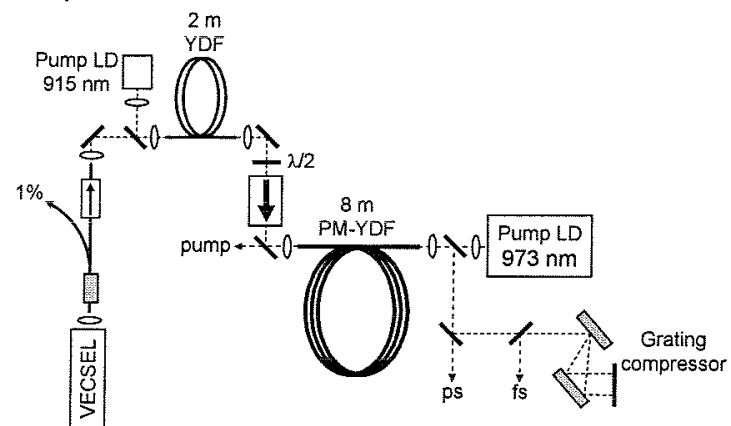

Figure 1: Schematic of the high repetition rate parabolic amplification system. LD: laser diode, YDF: ytterbium doped fiber, PM: polarization maintaining.

\section{Experimental results}

The final-stage power characteristic is shown in figure 2. A maximum output power of $53 \mathrm{~W}$ was achieved for $100 \mathrm{~W}$ of launched pump power corresponding to a maximum energy per pulse of $48 \mathrm{~nJ}$. The power evolution slightly differs from the linear trend due to the power dependence of the pump wavelength. The 
output beam quality factor was measured to be $M^{2} \sim 1.1$ ensuring nearly single-mode propagation in the high-power fiber amplifier.

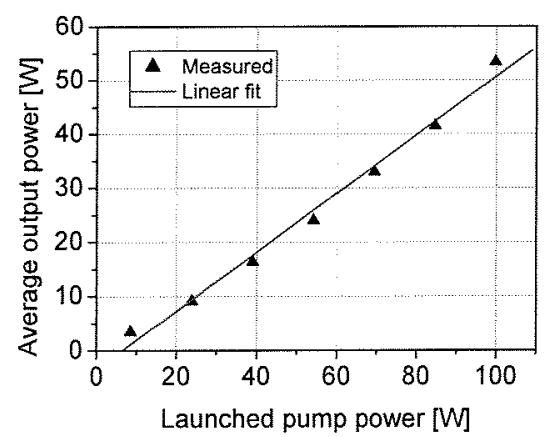

Figure 2: Output power vs. final-stage pump power. The input signal power was kept constant at $1.3 \mathrm{~W}$.

We observed temporal and spectral broadening of the output pulses, which is typical of self-similar parabolic amplification in normally dispersive fibers. Autocorrelation measurements of the output pulse width at full width half maximum (FWHM) reveal an increase from 2.2 ps to about 4.8 ps at minimum and maximum output power respectively. The 4.8 ps puises were compressed by a grating compressor down to an autocorrelation width of $170 \mathrm{fs}$. This corresponds to a puise duration of 110 fs FWHM assuming a sech ${ }^{2}$ pulse shape. The duty cycle becomes $10^{-4}$ and the time-bandwidth product 0.47 , close to the transform-limited value. Moreover the autocorrelation trace depicted in figure 3 emphasizes the excellent quality of compression with very little pedestal. This reveals the high linearity of the chirp before compression.

The output spectra measured at various average powers are also shown in the inset in figure 3 . Starting with a seed with spectral width of $2 \mathrm{~nm}$, the spectrum dramatically broadens to a bandwidth exceeding $15 \mathrm{~nm}$ FWHM at maximum power. The rapidly decreasing wings of the spectra are characteristic of parabolic amplification.

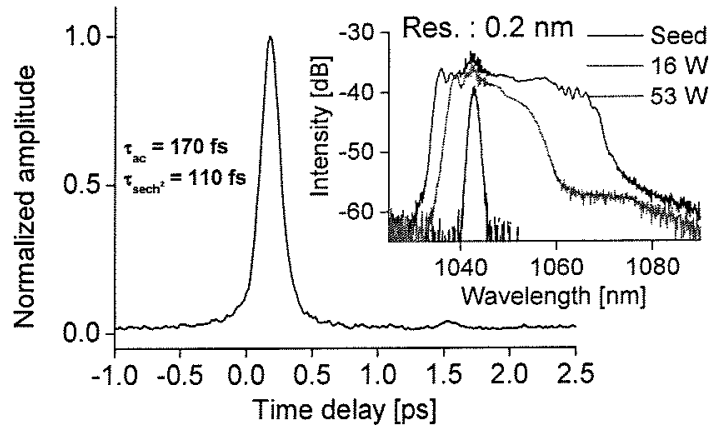

Figure 3: Autocorrelation trace of compressed pulse at maximum output power. Inset: output spectra at various output powers

This increase evolved asymmetrically with respect to the seed wavelength. This is explained by the reduced gain below $1035 \mathrm{~nm}$ and the high gain experienced by the signal at longer wavelengths. At higher output powers, highly distorted pulse shapes and spectra were observed possibly due to the truncated gain bandwidth at shorter wavelengths.

\section{Conclusions}

A femtosecond, $1.1 \mathrm{GHz}$ fiber MOPA system seeded by a passively mode-locked VECSEL has been demonstrated. A maximum output power of $53 \mathrm{~W}$ was reached. Although the seed wavelength was offcentered from the YDFA gain spectrum inducing asymmetric spectral broadening, excellent pulse compression down to 110 fs was achieved at maximum output energy of $48 \mathrm{~nJ}$. This result confirms the generation of parabolic pulses in the polarization maintaining fiber amplifier. Nevertheless the asymmetric behavior prevented further power scaling. This limiting factor as well as future directions will be discussed at the conference.

\section{References}

[1]K. Kurokawa et al., OFC 2006, PDP5

[2]M. E. Fermann et al., Phys. Rev. Lett. 84 (2000), 6010

[3] T. Schreiber et al., Opt. Lett. 31 (2006), 574

[4] A. Malinowski et al., Opt. Lett. 29 (2004), 2073

[5] P. Dupriez et al., CLEO 2006, CThJ3

[6] A. Garnache et al., Appl. Phys. Lett. 80 (2002), 3892

[7] V. I. Kruglov et al., J. Opt. Soc. Am. B. 19 (2002), 461 